\title{
Magnetic-Field-Enhanced Morphology of Tin Oxide Nanomaterials for Gas Sensing Applications
}

\author{
Jonathan C. Briones, Gwen Castillon, Michael P. Delmo, and Gil Nonato C. Santos
}

Physics Department, De La Salle University, 1004 Manila, Philippines

Correspondence should be addressed to Jonathan C. Briones; jonathan.briones@dlsu.edu.ph

Received 9 March 2017; Accepted 3 May 2017; Published 18 June 2017

Academic Editor: Ciyuan Qiu

Copyright ( 2017 Jonathan C. Briones et al. This is an open access article distributed under the Creative Commons Attribution License, which permits unrestricted use, distribution, and reproduction in any medium, provided the original work is properly cited.

\begin{abstract}
We studied the effect of an external magnetic field (up to $0.31 \mathrm{~T}$ ) on the growth of $\mathrm{SnO}_{2}$ nanowires fabricated using the horizontal vapor phase growth (HPVG) technique. The morphology of the nanowires was characterized by using scanning electron microscopy (SEM), and the chemical composition was characterized by energy dispersive X-ray (EDX) analysis. We found that the length of nanowires was significantly enhanced by the application of EMF. The aspect ratio, as well as the density of the fabricated nanowires, increased with increasing magnetic field intensity. Although the physics behind the morphology enhancement of the nanowires under magnetic field is still being investigated, nevertheless, we demonstrated that the magnetic field could be used as a key parameter to control the morphology of tin oxide nanomaterials grown via HPVG technique. The magnetically enhanced nanowires were used in the development of a gas sensor and were found to be sensitive to hydrogen sulfide gas and the headspace gas emitted by spoiling meat.
\end{abstract}

\section{Introduction}

There are tremendous interests in the fabrication of various nanomaterials with diversified morphologies, such as nanoparticles [1] and nanowires [2,3], because of their potential applications in electronics $[1,4,5]$ and medicine [6]. Physical and chemical $[4,7]$ methods, such as thermal evaporation [8], chemical vapor deposition [9], sol-gel method [10], and hydrothermal methods [8], are being used to fabricate nanomaterials. Typically, for the physical methods, temperature, pressure, and chemical addition [7] are the key parameters being controlled to modify the morphologies of nanomaterials. In this study, the parameter that was varied to control the morphology of tin oxide $\left(\mathrm{SnO}_{2}\right)$ nanomaterials fabricated by using our home-developed horizontal vapor phase growth (HVPG) technique [11] was the presence of an external magnetic field. The use of a magnetic field $(0.048 \mathrm{~T})$ as an external parameter in the HVPG method was first reported by De Mesa et al. [6] in the synthesis of iron oxide nanoparticles for glucose sensing applications which were claimed to have enhanced the superparamagnetic property of the material. No other attempts were made to fabricate nanomaterials using the HVPG technique with external magnetic field as a controlling parameter.

Nanostructured $\mathrm{SnO}_{2}$ offers a great potential for energy and environmental applications including gas sensing due to large surface area, low cost, and low toxicity [4]. Its gas sensing capabilities are widely reported due to its exceptionally high sensitivity to gas reaction and adsorption. Nonetheless, much innovative science needs discovery, and new techniques must be explored in the fabrication of this type of material by exploiting strategies in the field of materials science and nanotechnology. The use of chemical and biofunctionalized gas sensors is important in many aspects such as personal safety and security, detection and diagnosis of pollutants and poisons, health, semiconductor processing, agriculture, and automotive and aerospace industries $[2,12]$.

Recently, it has been shown that the HVPG deposition is successful in synthesizing nanostructured $\mathrm{SnO}_{2}$. However, the said technique allows the growth of many kinds of structures along the length of the substrate. Thus, understanding the growth mechanism of nanomaterials in HVPG technique 


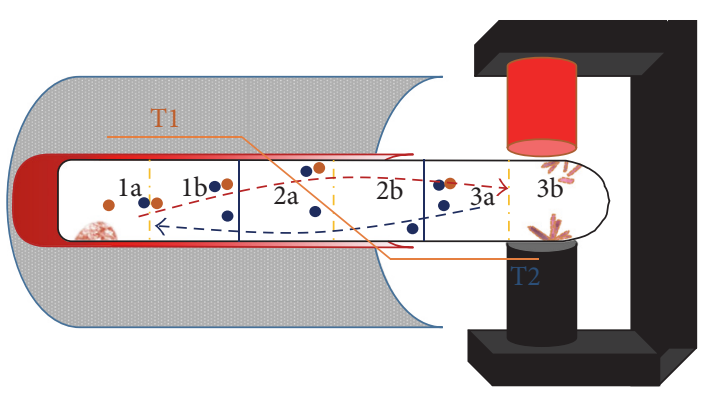

(a)

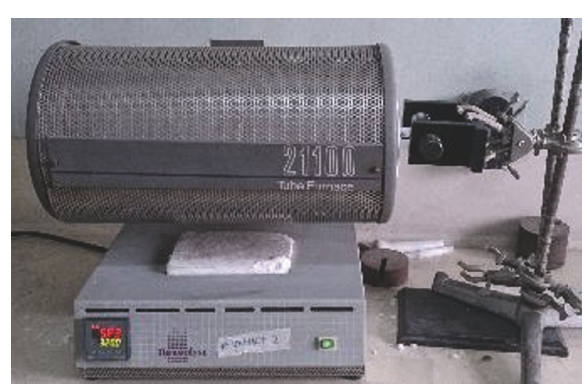

(b)

FIgURE 1: Horizontal vapor phase crystal growth setup. (a) Setup illustration. (b) Actual system.

would make it possible to control or alter the growth kinetics of the $\mathrm{SnO}_{2}$ nanomaterial resulting in better or improved materials and devices.

In this work, the effect of an external magnetic field ( 0 up to $0.31 \mathrm{~T}$ ) on the growth of tin oxide nanowires was examined. Magnetostatic interaction has an important role in the growth mechanism of nanomaterials. Several studies have reported on the influence of magnetic field on the nucleation kinetics of paramagnetic and diamagnetic materials during crystallization and precipitation. These reports verify the effect of applied magnetic field on the aspect ratio of nanostructures as well as its properties [13-15]. Applied magnetic field is cited to be an efficient tool in the fabrication of one-dimensional (1D) nanomaterials [16].

The fabrication of 1D metal oxide nanostructures, having diameters below $\sim 100 \mathrm{~nm}$, has attracted attention due to various applications in numerous fields. Due to the large surface-to-volume ratio in $1 \mathrm{D}$ structures like nanowires, nanorods, nanotubes, and so forth, a significant number of surface atoms (or molecules) can participate in surface reactions resulting in better sensitivity and selectivity $[2,3]$. One potential application of $1 \mathrm{D}$ structures is the evaluation of the freshness of meat through its headspace gas.

Meat spoilage is a sensorial quality consisting of the occurrence of off-odors (bacterial activity) and off-flavors or discoloration, which are characteristics that help determine its shelf life. Detectable organoleptic spoilage is a result of decomposition and the formation of metabolites caused by the growth of microorganisms. The emission of volatile gases by anaerobic and aerobic bacteria during spoilage can be used to detect the spoilage of meat [17]. Several studies have pointed to the concentrations of sulfurous compounds and biogenic amines as the primary factors that can be used in evaluating meat freshness. Thus, measuring the emission of volatile organic gases can be correlated to the degree of meat deterioration [18].

Sensory analysis of meat spoilage is very expensive and not always practical. Developing sensing materials that can ensure the safety and quality of meat products would benefit both producers and consumers of meat. The devices that need to be developed must not only be robust and compact but also have high sensitivity, selectivity, and reversibility in ambient temperature conditions with low manufacturing cost [12]. In an attempt to develop a device with the above-mentioned characteristics, the $\mathrm{SnO}_{2}$ nanostructures fabricated in this study were used as the sensing element of a spoilage detector gas sensor.

In this paper, external magnetic field intensities of $0 \mathrm{~T}$ (control), $0.25 \mathrm{~T}$, and $0.31 \mathrm{~T}$ were applied during the synthesis of tin oxide nanomaterials. The surface morphology and elemental composition were investigated using scanning electron microscopy (SEM), transmission electron microscopy (TEM), and energy dispersive X-ray (EDX) analysis, while the crystallinity was investigated using X-ray diffraction (XRD) analysis. The responses of the fabricated sensor substrate to hydrogen sulfide $\left(\mathrm{H}_{2} \mathrm{~S}\right)$ gas, ethanol, methanol, and meat headspace were also investigated.

\section{Experimental Setup}

2.1. Synthesis of Tin Oxide Nanomaterials via HVPG. Fused silica tubes with one end sealed were used as containers of the bulk $\mathrm{SnO}_{2}$ powder of $99 \%$ purity and $<5$-micron grain size obtained from Merck. The tubes were loaded with fifty (50) milligrams of bulk powder and then evacuated using a Thermionics High Vacuum System to a pressure of about $10^{-6}$ Torr. The tubes were then fully sealed using a high temperature blow torch to a length of $15 \mathrm{~cm}$.

The sealed tubes were inserted halfway through a Thermolyne horizontal tube furnace and then cured at $1,200^{\circ} \mathrm{C}$ with a ramp time of 40 minutes for 4-hour, 6-hour, and 8-hour curing time. Regions of interest were designated according to their respective position in the furnace during the annealing process. The part of the tube which contained the powder and which was inside the furnace was labeled section 1 . The middle portion of the tube near the opening of the furnace was designated as section 2 . The last region of interest, the part of the tube that was completely out of the furnace, was designated as section 3. Each section had a length of $5 \mathrm{~cm}$ and was further divided into two sections, $a$ and $b$. The experimental setup and schematic diagram are shown in Figure 1.

The position of the tube in the furnace provided a temperature gradient along its length. Using a type $\mathrm{K}$ thermocouple, it was confirmed that section 1 was heated at $1,200^{\circ} \mathrm{C}$. The temperature of section 2 was found to range from $353^{\circ}$ to $800^{\circ} \mathrm{C}$ along its length, while the temperature of section 3 was found to range from $63^{\circ}$ to $352^{\circ} \mathrm{C}$. 


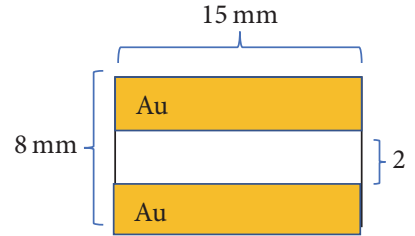

(a)

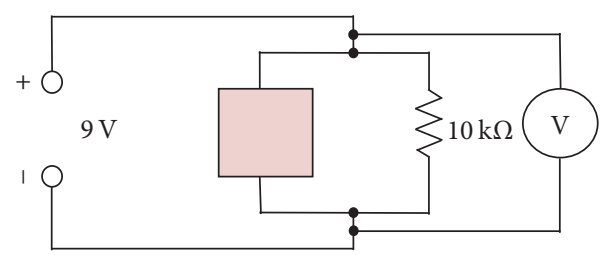

(b)

FIGURE 2: Schematic diagram of (a) an embodiment of a gas sensor electrode; (b) circuit diagram of gas sensor setup.

In order to verify the influence of the magnetic field on the growth of nanomaterials, its intensity was varied $(0 \mathrm{~T}, 0.25 \mathrm{~T}$, and $0.31 \mathrm{~T}$ ) as well as its position along the tube (sections 3a and $3 \mathrm{~b}$ ). The said magnetic field was supplied by a variable gap neodymium magnet.

JEOL-JSM 5310 Scanning Electron Microscope and Libra 200 CsSTEM Transmission Electron Microscope from Carl Zeiss Singapore were used in identifying the structures of the synthesized nanomaterials. Elemental composition of the samples was also determined using an Oxford EDX Link Isis System. The crystal structure of the as-synthesized nanomaterials was determined using Bruker D2 PHASER XRay Diffractometer.

2.2. Fabrication of the $\mathrm{SnO}_{2}$ Nanomaterial Sensor Substrate. The section with the highest density of synthesized nanowires was used in the fabrication of a gas sensing substrate. A $2 \mathrm{~mm}$ wide middle section of the collected substrates was then covered. To create gold electrodes on the substrates, the partly covered substrates were sputtered with gold for 30 seconds at $50 \mathrm{~mA}$ using a JEOL JFC-1200 Fine Coater. The resulting configuration of the gas sensing substrate is shown in Figure 2. The nanomaterials which were not sputtered with gold were the ones to detect gas emitted from spoiling meat as indicated by the change in the resistivity of the material, which was then converted to a voltage measurement and read via a PASCO Scientific voltage sensor.

A two-neck flat bottom flask was used as the chamber for testing the gas sensing ability of the sensor substrate. A Loadstar DC power supply was used to provide an input voltage of $9 \mathrm{~V}$ with current of $0.5 \mathrm{~A}$. A constant resistance provided by a $10 \mathrm{k} \Omega$ resistor was utilized (Figure 2(b)). Passport Interfaced PASCO Scientific voltage sensor and DataStudio for data acquisition were employed to measure the voltage response of the sensor (Figure 3). The experimental setup was operated at ambient temperature during the data acquisition.

\subsection{Nanomaterial Sensor Substrate Gas Sensing Test. To de-} termine the ability of the sensor substrate to detect gas and volatile compounds such as hydrogen sulfide $\left(\mathrm{H}_{2} \mathrm{~S}\right)$ gas, ethanol, methanol, and the headspace of confined fresh and spoiled pork meat samples were introduced into the gas chamber. The $\mathrm{H}_{2} \mathrm{~S}$ gas was prepared using $22 \mathrm{~g}$ of zinc sulfide $(\mathrm{ZnS})$ powder and $500 \mathrm{~mL}$ of $6 \mathrm{M}$ hydrochloric acid $(\mathrm{HCl})$. Meanwhile, the meat samples were allowed to spoil for 24 and 36 hours at room temperature. The response of the sensor substrate containing the synthesized nanowires was

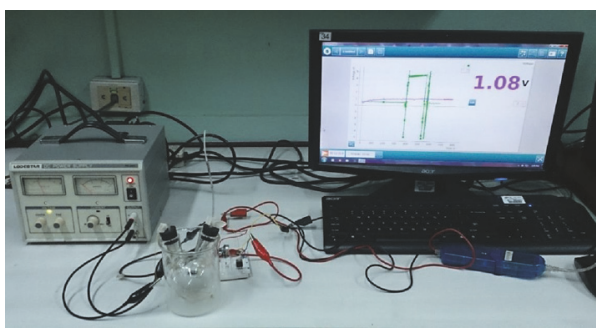

FIGURE 3: Experimental setup to test the gas sensing ability of the $\mathrm{SnO}_{2}$ sensor substrate.

compared to the response of the substrate with nanoparticles. This is to check the sensing enhancement brought about by the $1 \mathrm{D}$ structure.

The headspace gas introduced to the sensor substrate was characterized by gas chromatography-mass spectrometry (GC-MS) using a 50/30 um DVB/carboxen/PDMS coating SPME fiber to absorb the gas introduced to the sensor substrate. Five (5) grams of the meat sample was placed into a flat bottom headspace vial fitted with a septum and crimp cap. The vial was heated using a water bath at $80 \mathrm{deg}$ for 5 minutes. The SPME fiber $(50 / 30 \mu \mathrm{m} \mathrm{DVB/carboxen/PDMS}$ coating) was preconditioned prior to analysis at $240^{\circ} \mathrm{C}$ for 30 min. After extraction, the SPME fiber was removed from the headspace vial and inserted directly at the injection port of the gas chromatograph. The PerkinElmer Clarus 500 Gas Chromatograph-Mass Spectrometer equipped with MS fused silica capillary column was used. The pressure was set at $43 \mathrm{~Pa}$, with flow rate of $7 \mathrm{~mL} / \mathrm{min}$. Chromatographic conditions included oven temperature of $40^{\circ} \mathrm{C}$ for $2 \mathrm{~min}$ which was later increased at a rate of $5^{\circ} \mathrm{C} / \mathrm{min}$ to $240^{\circ} \mathrm{C}$. The mass spectrometer was used in electron impact mode with a source temperature of $200^{\circ} \mathrm{C}$, ionizing voltage of $70 \mathrm{eV}$, and transfer line of $240^{\circ} \mathrm{C}$. Peak identification was determined by comparison of volatile sample mass spectra with spectra in NIST/EPA/NIH Mass Spectral Database (Nat. Institute of Standards and Tech.).

\section{Results and Discussion}

The growth mechanism of $\mathrm{SnO}_{2}$ nanomaterials via the HVPG technique is attributed to the vapor-liquid-solid (VLS) mechanism. The results suggested that, during the crystal growth, the $\mathrm{SnO}_{2}$ source material, primarily located in section 1 , sublimes to vapor phase and was transported to the different 


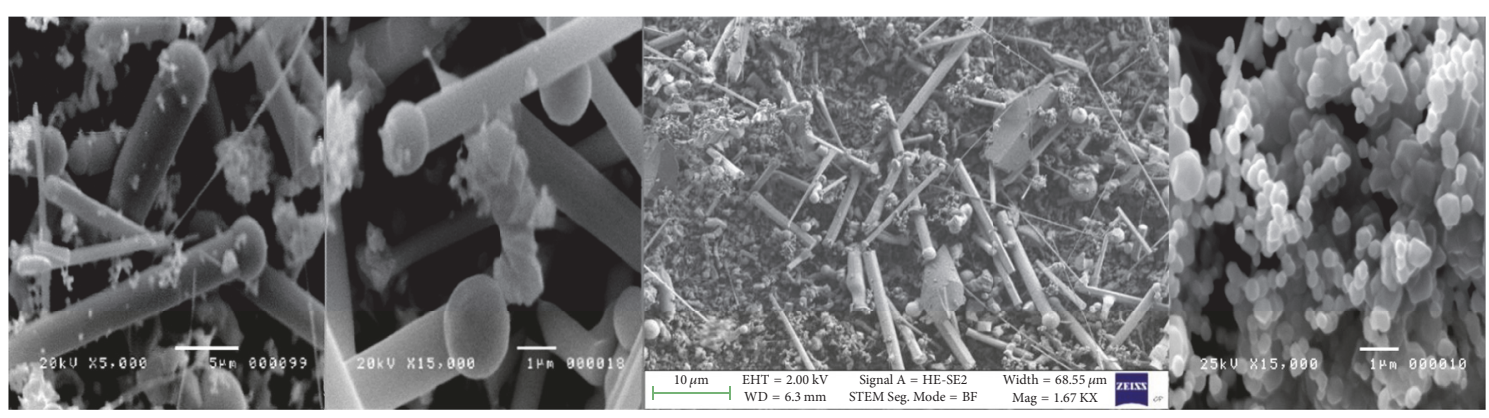

(a)

(b)

(c)

FIGURE 4: Micrographs showing the intermediate step in the formation of nanorods with spherical tips indicating their growth dynamics: (a) SEM (b) TEM, and (c) nanoparticles grown via the HVPG technique.

zones in the tube, mostly to the cooler regions of sections 2 and 3. The convective motion of the gas inside the quartz tube was greatly influenced by the thermal gradient existing along the length of the tube.

One possibility is that as the vapor moved towards section 3 , it encountered the colder region section 2 , where it could have undergone a phase transition from gas to liquid. It is also possible that some of the vapor interacted with the walls of the tube upon reaching section 3 and lost some of its energy, thereby condensing into liquid Sn in the process. The $\mathrm{SnO}$ vapor would have condensed first to liquid state in section 2 and then section 3 together with the liquid Sn metal. This is observed in the intermediate step formation of nanorods (with spherical tips) in section 2 as shown in Figure 4. The Sn metal may have acted as a catalyst to assist the growth of one-dimensional structure, forming a liquid with a component of the solid phase, giving way for the formation of $\mathrm{SnO}_{2}$ nanowires and nanorods $[1,4]$. Following the periods of nucleation and incubation, a crystallite will commonly develop into a three-dimensional object with well-defined low index crystallographic faces. The growth process eventually terminates when the temperature is below the melting point, conforming to sections 2 and 3 temperatures. $\mathrm{SnO}_{2}$ is reported to crystallize with a tetragonal rutile structure (cassiterite) wherein the tin atoms are 6 coordinates and the oxygen atoms are three coordinates [4, $7,19]$.

In the absence of applied magnetic field $(0 \mathrm{~T})$, the diameters of the grown nanoparticles were found to increase with increasing curing time. However, there were not any nanowires formed on the substrate. With the presence of magnetic field, nanowire formation with diameter less than $100 \mathrm{~nm}$ was favored with a more defined structure when the magnet is farther from the furnace or at section $3 \mathrm{~b}$, and at greater intensity $(0.31 \mathrm{~T})$. Notably, a strong magnetic field intensity greatly improved the tractability of the growth of nanowires. In addition, the density of the nanowires was observed to increase in longer curing time, 8 hours, as shown in Figure 5.

On the other hand, lower magnetic field intensity, $0.25 \mathrm{~T}$, was observed to produce a lower yield of nanomaterials. The length of the synthesized nanomaterials was also observed to be shorter than those produced under the influence of a higher magnetic field intensity. A comparison of the synthesized nanostructures at varying positions where the magnetic field was applied (section 3a or section $3 \mathrm{~b}$ ), varying magnetic field intensities, and varying curing times is shown in Figure 5. It can be seen from Figure 5 that the yield of nanowires is greater when the magnet is positioned at section $3 \mathrm{~b}$, where the temperature is relatively cooler than that of section $3 \mathrm{a}$.

To verify the influence of magnetic field on the growth of $\mathrm{SnO}_{2}$ nanowires, the deposited structures on adjacent substrate sections ( $3 \mathrm{a}$ and $3 \mathrm{~b}$ ) were compared. As shown in Figure 6(a), nanoparticles and orthorhombic structures were grown in section $3 a$ without an applied magnetic field. It is clear that there are no nanowires grown on the substrate. Meanwhile, in the adjacent section applied with magnetic field, high yield of nanowires was synthesized (Figure 6(b)). On the other hand, nanowires were found in section 3a applied with magnetic field (Figure 6(c)) whereas no nanowires were seen in the adjacent section (3b) without applied field (Figure 6(d)). Similarly, for the same substrate section applied with $0 \mathrm{~T}$ (no external magnetic field), $0.25 \mathrm{~T}$, and $0.31 \mathrm{~T}$ magnetic field intensity, nanowires were only seen in the latter two setups. This meant that the presence of the magnetic field influenced the growth of $\mathrm{SnO}_{2}$ nanowires.

The nanowires formed under the influence of magnetic field were found to have higher aspect ratio at higher magnetic field intensity $(0.31 \mathrm{~T})$ as illustrated in Figure 7 . At the same magnetic field intensity, higher aspect ratio was computed when the substrate is farther from the furnace or at section $3 \mathrm{~b}$ of the reaction tube. This means that nanowires with smaller diameter and longer lengths were formed under these conditions.

The formation of the nanowires is believed to be due to the presence of the external magnetic field, along with the presence of temperature gradient. The magnetic field exposure might have retarded the nucleation rate and accelerated the crystal growth favoring nanowire formation [13]. The particles interconnected with each other eventually forming the nanowire-like formation. The morphology enhancement of the nanowires under magnetic field is suspected to be due to thermoelectric magnetohydrodynamics as the flux 
(a)

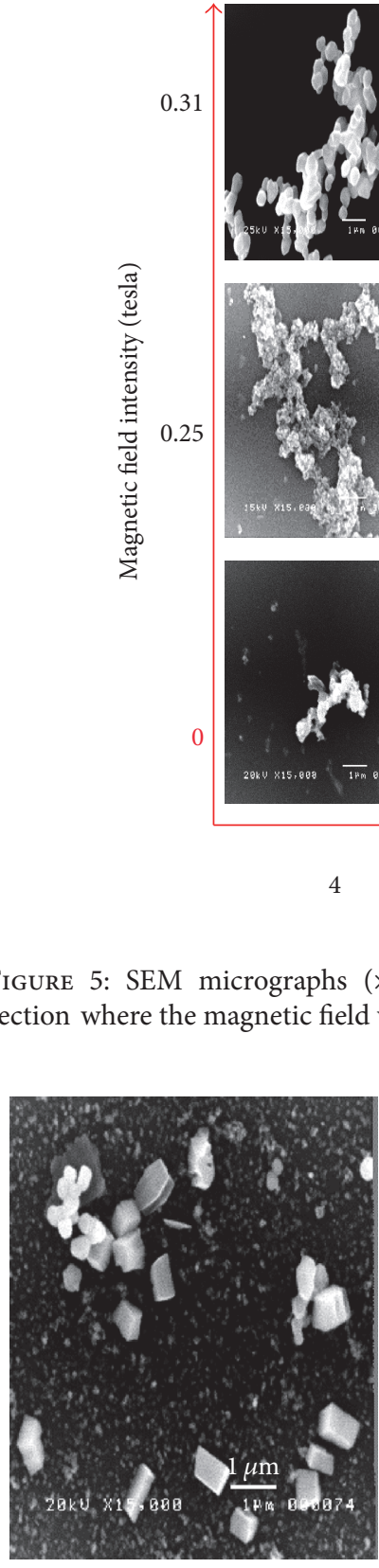

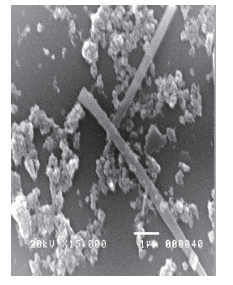
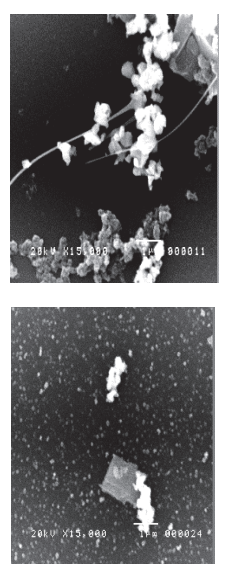

$3 a$
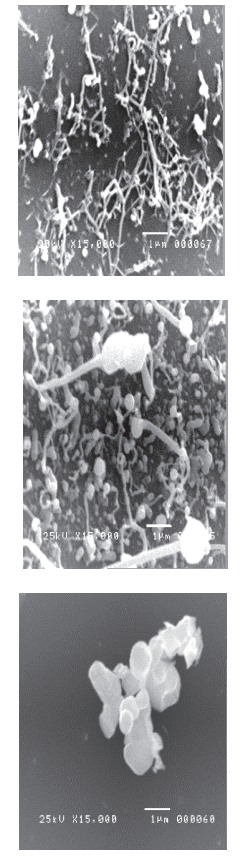

8
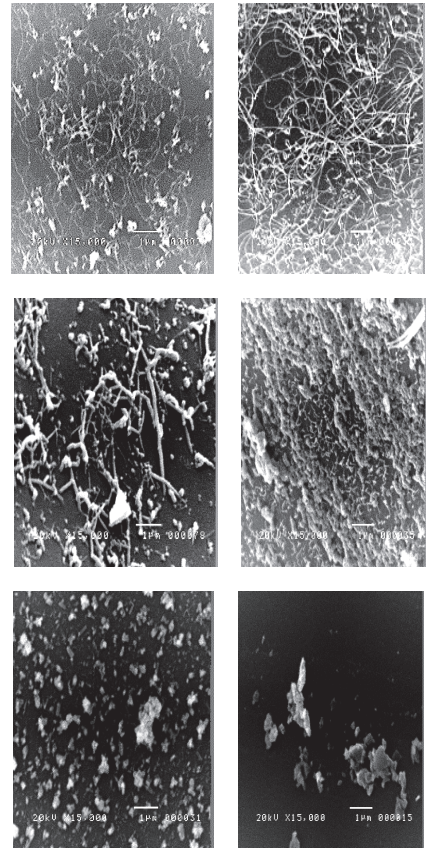

$3 \mathrm{~b}$
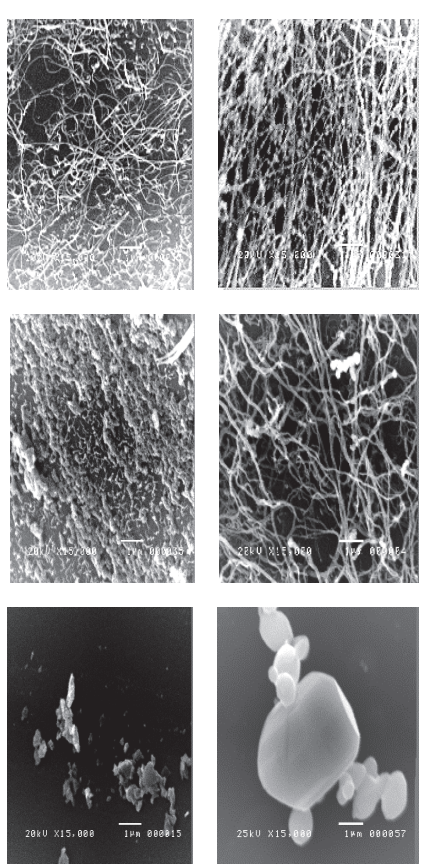

8

Time (h)

FIGURE 5: SEM micrographs $(\times 15,000)$ comparing the nanostructures formed under different magnetic field intensities, substrate section where the magnetic field was applied, and curing time.

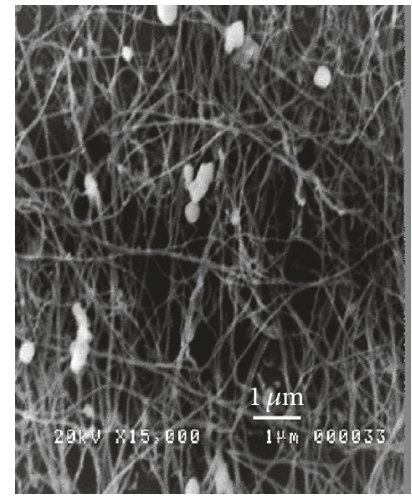

(b)

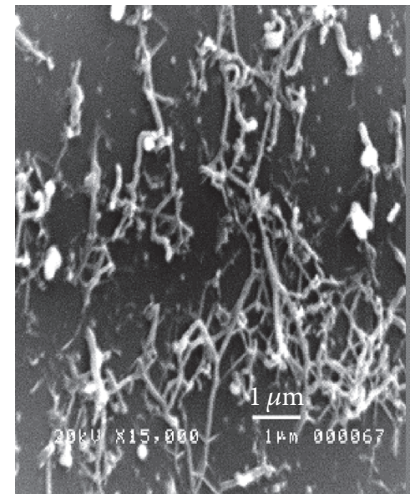

(c)

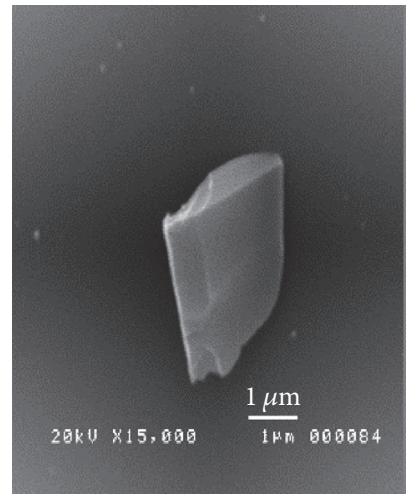

(d)

FIGURE 6: Synthesized nanostructures in adjacent sections of the substrates: (a) absence of magnet on section 3a; (b) presence of magnet on section $3 \mathrm{~b}$ and adjacent sections, (c) presence of magnet on 3a; (d) absence of magnet on $3 \mathrm{~b}$.

of vaporized material interacts with the magnetic lines of force together with the presence of a temperature gradient. However, this needs further investigation.

Tin oxide is a wide gap $(3.6 \mathrm{eV}) \mathrm{n}$-type semiconductor. Reports have stated a high Seebeck coefficient $\left(185.38 \mu \mathrm{V} /{ }^{\circ} \mathrm{C}\right)$ of tin oxide thin film deposited glass substrates. Even higher coefficients were obtained when the samples were deposited with metal films such as $\mathrm{Ag}\left(225.24 \mu \mathrm{V} /{ }^{\circ} \mathrm{C}\right)$ and $\mathrm{Cr}$ $\left(323.68 \mu \mathrm{V} /{ }^{\circ} \mathrm{C}\right)[20]$. It can also be noted that other factors, such as the epitaxial orientation defined by the substrate, have an influence on the growth direction. EDX spectroscopy confirms the presence of tin and oxygen for the tin oxide samples as shown in Figure 8.

Figure 9 exhibits the XRD pattern of the $\mathrm{SnO}_{2}$ bulk powder source material and $\mathrm{SnO}_{2}$ nanomaterial grown at $1200^{\circ} \mathrm{C}$ using the HVPG method, applied with $0.31 \mathrm{~T}$ external magnetic field. The $\mathrm{SnO}_{2}$ powder has diffraction peaks at (110), (101), (200), and (211) planes which can be readily indexed with a tetragonal rutile structure with lattice con-

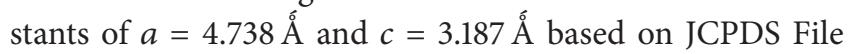




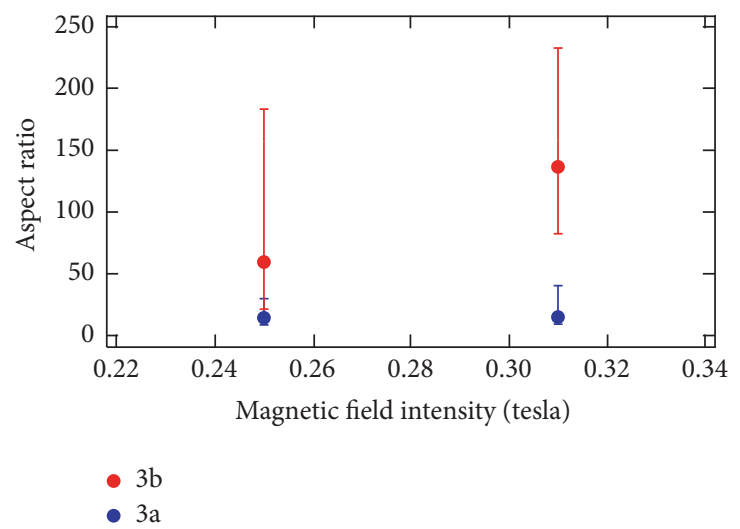

FIGURE 7: Aspect ratio of the nanowires grown under different magnetic field intensities and positions in the tube.

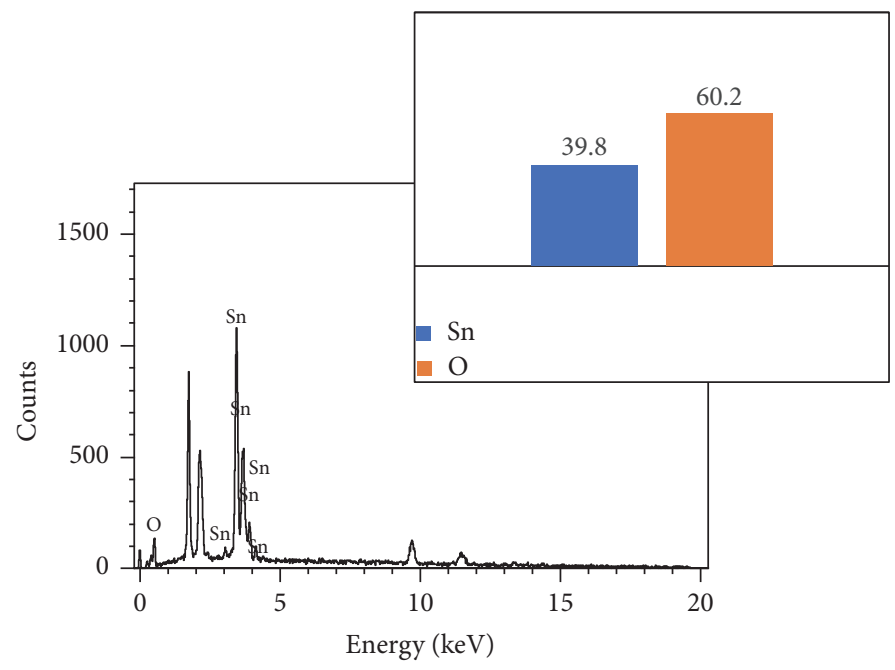

FIGURE 8: Energy dispersive X-ray spectrum of the sample indicating the presence of tin and oxygen and their atomic ratio of approximately $1: 2$.

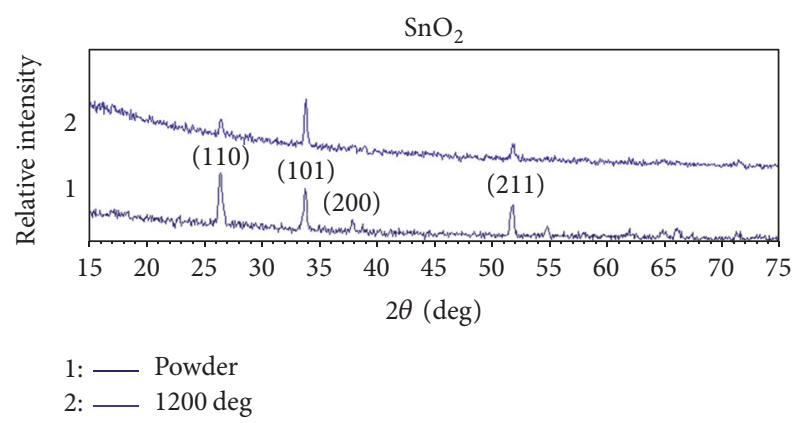

FIGURE 9: XRD pattern of $\mathrm{SnO}_{2}$ bulk powder and $\mathrm{SnO}_{2}$ synthesized nanowires.

number 41-1445 while the $\mathrm{SnO}_{2}$ nanomaterial has similar diffraction peaks but low intensity peaks. This may be due to the crystallite size variations.

To determine the ability of the gas sensor substrate to detect the presence of gas analytes, the headspace gas produced by meat inside the test tube was introduced into the glass chamber. The voltage response of the sensor substrate with the synthesized nanowires was recorded for each setup as shown in Figure 10. The said response was then compared to a sensor substrate with nanoparticles (Figure 11).

No significant change in the voltage measurements was measured upon the introduction of the fresh meat headspace (Figure 10(a)). On the other hand, an increase in the voltage reading of nearly twice the value as the baseline voltage was recorded with the introduction of the $24 \mathrm{~h}$ spoiled meat headspace into the chamber. As shown in Figure 10(b), the voltage measurement increased from $1.26 \mathrm{~V}$ to $2.39 \mathrm{~V}$. A similar behavior was observed upon the introduction of the $36 \mathrm{~h}$ spoiled meat headspace into the chamber but with a slightly higher change in the measurement, from $1.67 \mathrm{~V}$ to $3.28 \mathrm{~V}$ (Figure 10(c)).

The meat headspace was also tested in a sensor substrate with different $\mathrm{SnO}_{2}$ nanomaterial morphology. The nanoparticles synthesized in the substrate without external magnetic field application were used as a sensing material in the same gas extracts. Similar to the nanowires counterpart, no significant change in the voltage measurement was recorded for the fresh meat headspace; however, the baseline was found to be noisy or oscillating (Figure 11(a)). Introduction of the 


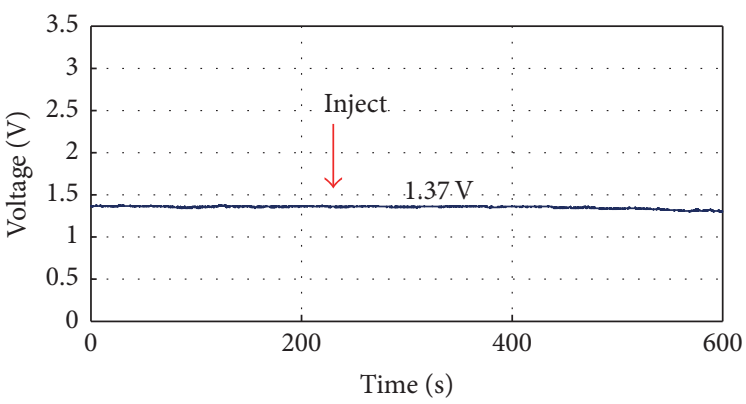

(a)

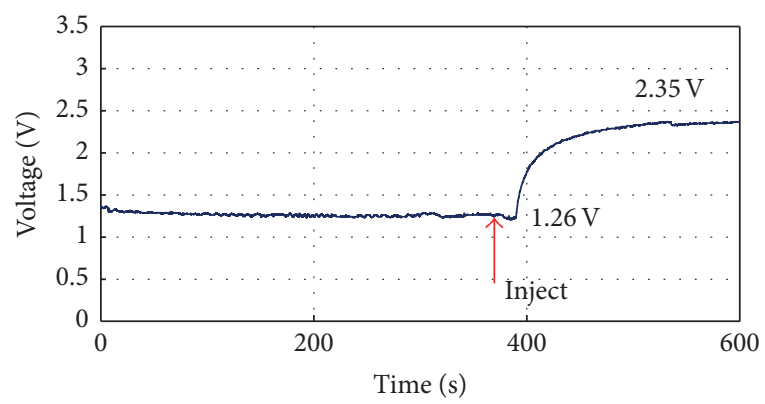

(b)

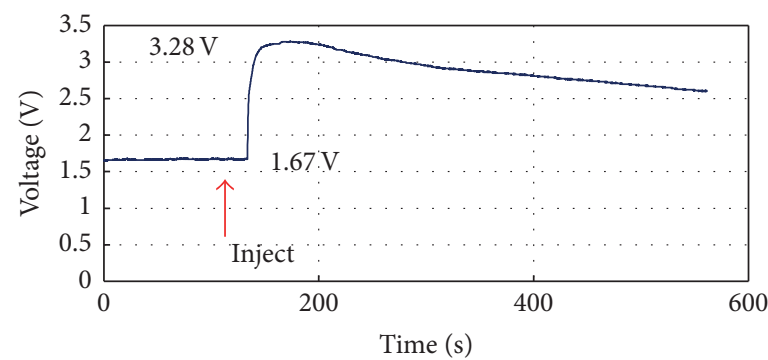

(c)

FIGURE 10: Results obtained when the gas sensing device that includes the synthesized $\mathrm{SnO}_{2}$ nanowires electrode was used to detect headspace gas: (a) fresh meat, (b) $24 \mathrm{~h}$ spoiled meat, and (c) $36 \mathrm{~h}$ spoiled meat.

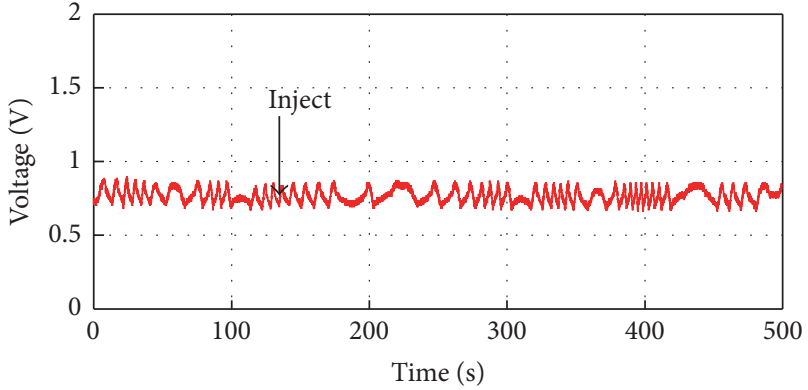

(a)

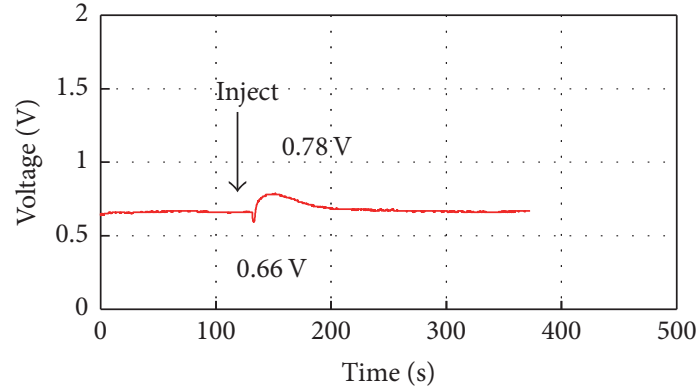

(b)

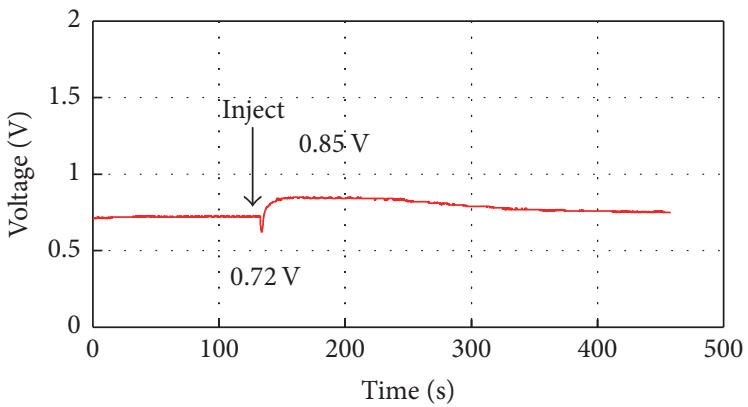

(c)

FIGURE 11: Results obtained when the gas sensing device that includes the $\mathrm{SnO}_{2}$ nanoparticles electrode was used to detect headspace gas: (a) fresh meat, (b) $24 \mathrm{~h}$ spoiled meat, and (c) $36 \mathrm{~h}$ spoiled meat.

24-hour and 36-hour spoiled meat headspace resulted in an increase in the voltage measurement. The said increase in the voltage measurement for the 24-hour and 36-hour headspace was found to be relatively identical and smaller than the nanowire sensor response. The difference in the signal of the former to the latter could be attributed to the higher aspect ratio of the nanowires which resulted in a greater sensitivity.

In order to determine the possible compound to which the $\mathrm{SnO}_{2}$ sensor substrate responds, both fresh and spoiled meat samples were subjected to headspace solid 


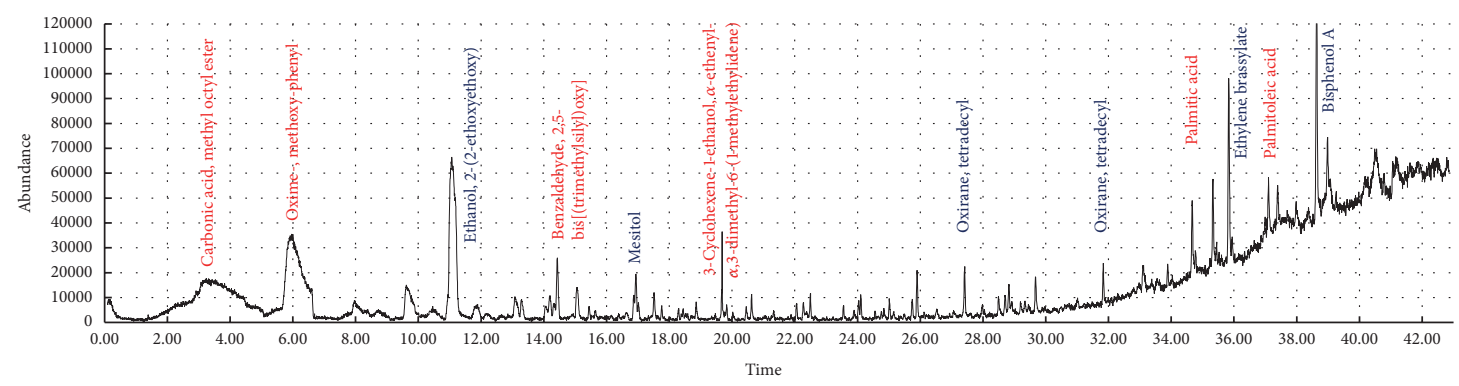

FIGURE 12: GC-MS chromatogram of fresh pork meat headspace. The elevation of the spectrum on the right is due to the high source temperature.

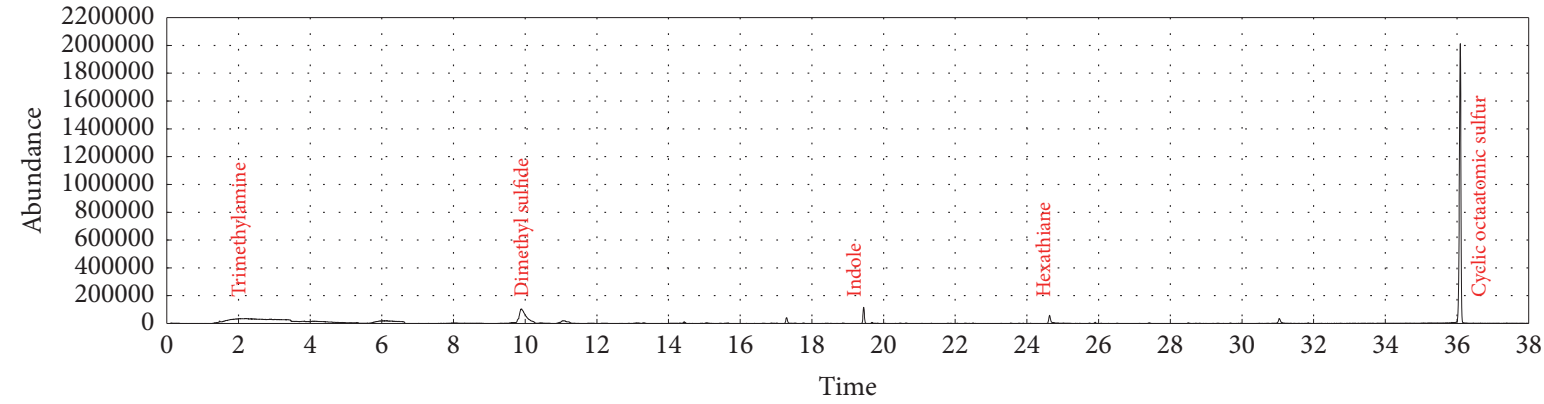

FIGURE 13: GC-MS chromatogram of $36 \mathrm{~h}$ spoiled pork meat headspace.

phase microextraction-gas chromatography-mass spectrometry (SPME-GC-MS). This was done to analyze the volatile compounds present in the headspace of the samples and to discriminate the gas that the fabricated sensor substrate responds to.

Mass spectrometry of the fresh meat headspace revealed strong sharp peaks for benzaldehyde, 2,5-bis[(trimethylsilyl)oxy]; 3-cyclohexene-1-ethanol, $\alpha$-ethenyl- $\alpha$,3-dimethyl6-(1-methylethylidene); and n-hexadecanoic acid (palmitic acid) as shown in Figure 12. Other compounds identified include palmitoleic acid; carbonic acid; methyl octyl ester; and oxime-, methoxy-phenyl. The sensor substrate did not show significant response to the headspace of the fresh sample and thus to its constituent compounds.

Headspace of spoiled pork was found to be largely composed of sulfur compounds with the highest abundance for cyclic octaatomic sulfur (Figure 13). This compound and hexathiane (hexasulfur) are identified to be produced by some strain of Pseudomonas (Pseudomonas spp.) and are associated with the foul odor in the spoiled meat samples $[21,22]$. Aminomethanesulfonic acid and benzaldehyde, 2,5bis [(trimethylsilyl)oxy] were also found in the samples. Other volatile compounds, in lesser abundance, identified in the pork headspace were indole (foul odor associated with spoiling meat originating from tryptophan), dimethyl sulfide (offodor produced by organisms like $P$. fragi, B. thermosphacta, and S. putrefaciens), dimethyl trisulfide, and trimethylamine (a product of decomposition) [21].

With the sensor substrate's recorded response to the headspace of the spoiled meat, it is possible that the said sensor is responding to the sulfur compounds which were found

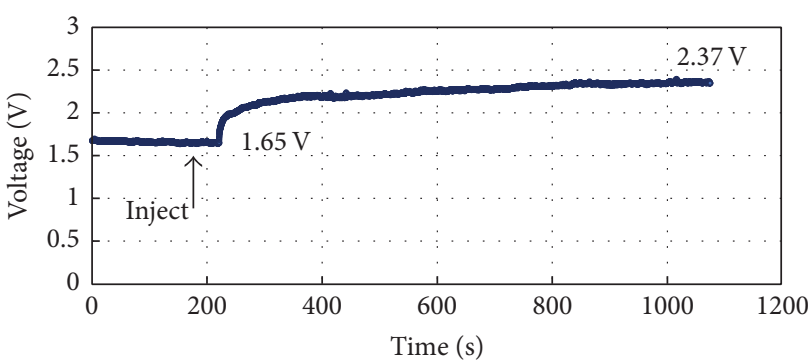

FIGURE 14: Results obtained when the gas sensing device that includes $\mathrm{SnO}_{2}$ nanowires sensor electrode was used to detect $\mathrm{H}_{2} \mathrm{~S}$.

to increase with increasing time of spoilage. A reference test was then conducted using hydrogen sulfide $\left(\mathrm{H}_{2} \mathrm{~S}\right)$ as the gas analyte.

Similar to the response of the sensor substrate to the headspace of spoiled meat, an increase in voltage measurement of about $0.7 \mathrm{~V}$ was recorded with the introduction of $1 \mathrm{~cm}^{3}$ of $\mathrm{H}_{2} \mathrm{~S}$ gas into the chamber (Figure 14). Succeeding trials showed the same pattern of response. Compared to the sensor substrate of a different morphology, the nanoparticle sensor gives out a small increase in voltage as compared to the synthesized nanowires counterpart (Figure 15). The difference in the signal of the nanowire sensor to the nanoparticle sensor could be attributed to the higher aspect ratio of the former leading to its sensitivity enhancement.

The sensor substrates' response to ethanol and methanol vapors was also explored. Ethanol and methanol are among the volatile compounds produced from bacterial metabolism 


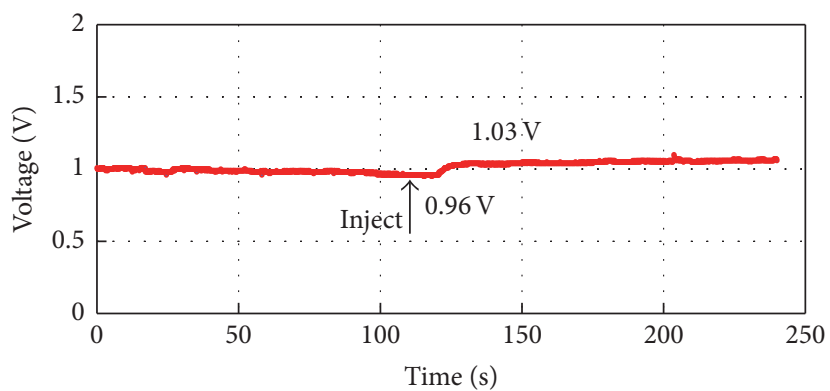

FIGURE 15: Results obtained when the gas sensing device that includes $\mathrm{SnO}_{2}$ nanoparticles sensor electrode was used to detect $\mathrm{H}_{2} \mathrm{~S}$.

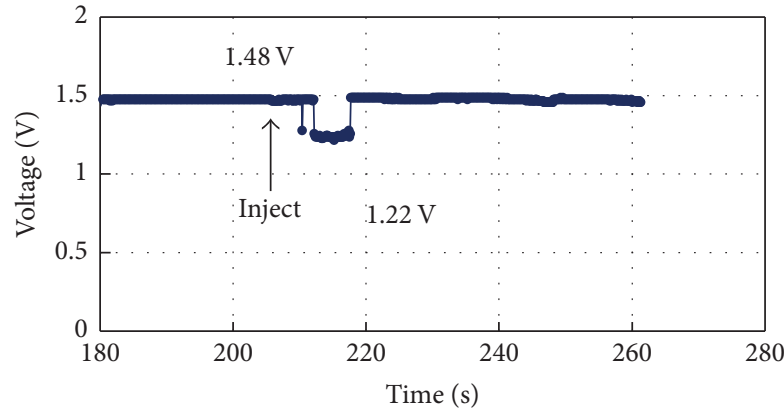

(a)

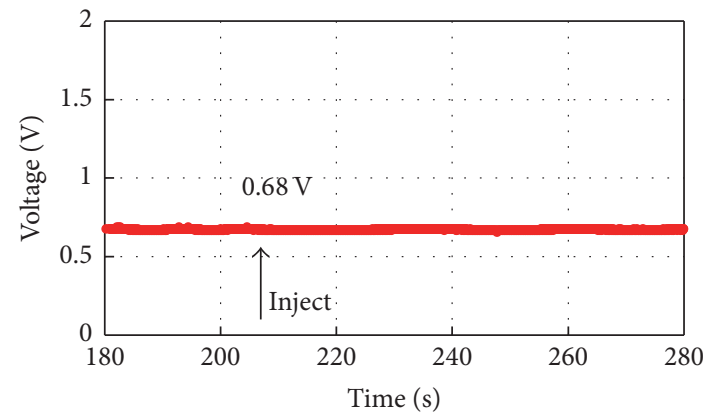

(b)

Figure 16: Results obtained when the gas sensing device that includes $\mathrm{SnO}_{2}$ gas sensor electrode was used to detect ethanol vapor: (a) nanowires, (b) nanoparticles.

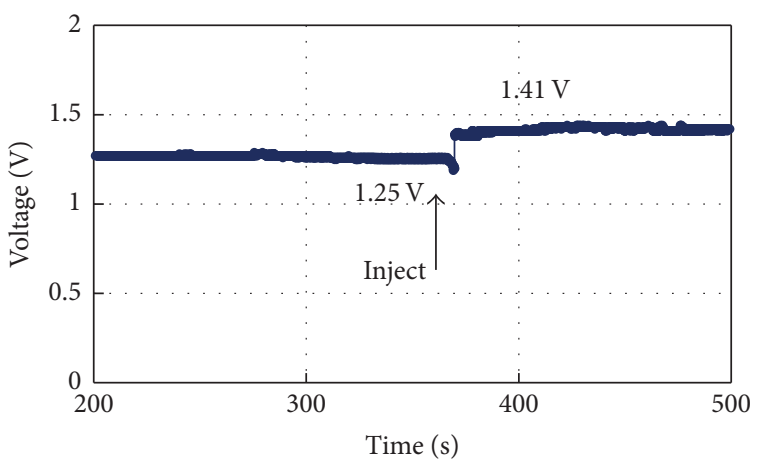

(a)

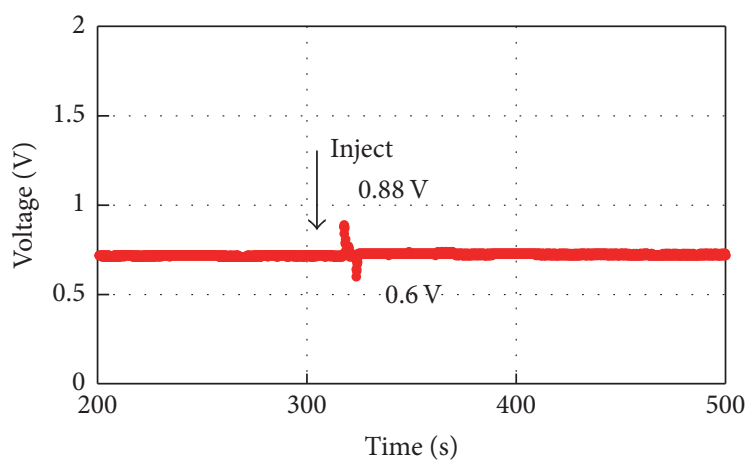

(b)

FIGURE 17: Results obtained when the gas sensing device that includes $\mathrm{SnO}_{2}$ gas sensor electrode was used to detect methanol: (a) nanowires, (b) nanoparticles.

in meat $[21,23,24]$. A decrease in the voltage measurement was observed upon the introduction of the ethanol vapor to the synthesized nanowire sensor substrate (Figure 16(a)); meanwhile, no significant response was recorded for the nanoparticle sensor substrate. Ethanol is reported to be a reducing gas which could possibly explain the decrease in resistance or the voltage reading in the sensor substrate [25].

On the other hand, a successive decrease and increase in the voltage measurement were noted for the nanowire sensor while an increase and then a decrease in reading were observed in the nanoparticles sensor substrate upon the introduction of methanol gas (Figure 17). Corresponding results were observed in the repeated trials.

The trapping of electrons at adsorbed molecules and band bending induced by these charged molecules are identified as the reasons for the change in the resistivity. The sensing reaction to the $\mathrm{H}_{2} \mathrm{~S}$ gas or the sulfur compounds found in the meat headspace is believed to occur only on the surface of the sensing material. The application of magnetic field to the synthesis of nanomaterials could have increased its defects, that is, oxygen vacancy which may have resulted in improved sensitivity. A charge depletion layer can be formed 
near the grain surface of $\mathrm{SnO}_{2}$ in an oxidizing atmosphere. The electrons associated with these are drawn from the conduction band of the material leading to an increase in resistance. Given a constant current, this results in the increase in voltage measurement $[5,26]$.

\section{Conclusions}

The presence of the magnetic field, along with the presence of temperature gradient, was found to be responsible for the formation of the homogenized nanowires. However, the temperature gradient is believed to be a primary influence as compared to the applied magnetic field intensity on the growth of the nanowire. We found that length of nanowires was significantly enhanced by the application of magnetic field. The aspect ratio, as well as the density of the synthesized nanowires, increased with increasing magnetic field. Thermoelectric magnetohydrodynamics is suspected to be the mechanism for the formation of nanowires but this needs to be validated.

As a proof of concept, the fabricated $\mathrm{SnO}_{2}$ sensor substrate was found to be more responsive to gaseous sulfur compounds and has potential application in meat spoilage evaluation and $\mathrm{H}_{2} \mathrm{~S}$ detection.

\section{Conflicts of Interest}

The authors declare that there are no conflicts of interest regarding the publication of this paper.

\section{Acknowledgments}

The authors would like to express their appreciation to the i-Nano Lab graduate students, staff of the Surface Physics Laboratory, the DLSU College of Science, the DLSU VCRI office, the DOST-SEI-ASTHRDP, and Carl Zeiss Singapore for the TEM micrographs.

\section{References}

[1] R. De Los and G. N. Santos, "Growth mechanism of $\mathrm{SnO}_{2}$ nanomaterials derived from backscattered electron image and Edx observations," International Journal of Scientific \& Engineering Research, vol. 2, no. 12, 2011.

[2] A. Kolmakov and M. Moskovits, "Chemical sensing and catalysis by one-dimensional metal-oxide nanostructures," Annual Review of Materials Research, vol. 34, pp. 151-180, 2004.

[3] E.-S. M. A. Duraia, Z. A. Mansorov, and S. Tokmolden, "Synthesis, characterization and photoluminescence of tin oxide nanoribbons and nanowires," Physica B: Condensed Matter, vol. 404, no. 21, pp. 3952-3956, 2009.

[4] S. Das and V. Jayaraman, " $\mathrm{SnO}_{2}$ : a comprehensive review on structures and gas sensors," Progress in Materials Science, vol. 66, pp. 112-255, 2014.

[5] M. Batzill and U. Diebold, "The surface and materials science of tin oxide," Progress in Surface Science, vol. 79, no. 2-4, pp. 47-154, 2005.

[6] D. M. De Mesa, G. N. Santos, and R. V. Quiroga, "Synthesis and characterization of $\mathrm{Fe}_{2} \mathrm{O}_{3}$ nanomaterials using Hvpc growth technique for glucose sensing application," International Journal of Scientific \& Engineering Research, vol. 3, no. 8, 2012.

[7] G. Cao, Nanostructures \& Nanomaterials: Synthesis, Properties \& Applications, Imperial College Press, 2004.

[8] A. Johari, M. C. Bhatnagar, and V. Rana, "Growth, characterization and I-V characteristics of tin oxide $\left(\mathrm{SnO}_{2}\right)$ nanowires," Advanced Materials Letters, vol. 3, no. 6, pp. 515-518, 2012.

[9] M. Salavati-Niasari, N. Mir, and F. Davar, "Synthesis, characterization and optical properties of tin oxide nanoclusters prepared from a novel precursor via thermal decomposition route," Inorganica Chimica Acta, vol. 363, no. 8, pp. 1719-1726, 2010.

[10] M. A. Batal, G. J. Nashed, and J. Fares Haj, "Electrical properties of nanostructure tin oxide thin film doped with copper prepared by Sol-Gel method," Latin-American Journal of Physics Education, vol. 6, no. 2, pp. 311-316, 2012.

[11] G. N. C. Santos, A. A. Salvador, and R. V. Quiroga, “Temperature and deposition time dependence of the geometrical properties of tin oxide nanostructures," International Journal of Scientific \& Engineering Research, vol. 2, no. 10, 2011.

[12] G. Jiménez-Cadena, J. Riu, and F. X. Rius, "Gas sensors based on nanostructured materials," Analyst, vol. 132, pp. 1083-1099, 2007.

[13] T. R. Bastami and M. H. Entezari, "Synthesis of manganese oxide nanocrystal by ultrasonic bath: effect of external magnetic field," Ultrasonics Sonochemistry, vol. 19, no. 4, pp. 830-840, 2012.

[14] A. B. Freitas, F. G. Landgraf, M. M. Seckler, and M. Giulietti, "The influence of magnetic field on crystallization from solutions," in Proceedings of the International Conference of Industrial Crystallization, Cambridge, UK, September 1999.

[15] M. Saeidi, M. Vaezzadeh, and M. Mansouri, "Influence of AC magnetic field on phonon vibrations of superparamagnetic cluster sitting at tip end of ultra-long carbon nanotube," Journal of Crystal Growth, vol. 345, no. 1, pp. 7-10, 2012.

[16] R. P. Ji, J. S. Jiang, and M. Hu, "How does the distribution of external magnetic lines of force influence the growth of ferromagnetic material?" Journal of Physical Chemistry, vol. 114, no. 28, pp. 12090-12094, 2010.

[17] N. El Barbri, E. Llobet, N. El Bari, X. Correig, and B. Bouchikhi, "Electronic nose based on metal oxide semiconductor sensors as an alternative technique for the spoilage classification of red meat," Sensors, vol. 8, no. 1, pp. 142-156, 2008.

[18] Z. Li and K. Suslick, "Portable optoelectronic nose for monitoring meat freshness," ACS Sensors, vol. 1, no. 11, pp. 1330-1335, 2016.

[19] A. Ansari, P. Solanki, A. Kaushik, and B. Malhotra, "Recent advances in nano- structured metal oxides based electrochemical biosensors for clinical diagnostics," in Nanostructured Materials for electrochemical biosensors, NOVA, New York, NY, USA, 2009.

[20] S. Anuradha and K. Rajanna, "A novel method for the improvement in thermoelectric property of tin oxide thin films and its application in gas sensing," International Journal on Smart Sensing and Intelligent Systems, vol. 1, no. 2, pp. 498-511, 2008.

[21] J. Jay, Modern Food Microbiology, Aspen Publication, Frederick, Md, USA, 6th edition, 2000.

[22] D. Mayr, R. Margesin, E. Klingsbichel et al., "Rapid detection of meat spoilage by measuring volatile organic compounds by using proton transfer reaction mass spectrometry," Applied and Environmental Microbiology, vol. 69, no. 8, pp. 4697-4705, 2003. 
[23] A. Argyri, A. Mallouchos, E. Panagou, and G. Nychas, "The dynamics of the HS/SPME-GC/MS as a tool to assess the spoilage of minced beef stored under different packaging and temperature conditions," International Journal of Food Microbiology, vol. 193, pp. 51-58, 2015.

[24] F. Leroy, C. Vasilopoulus, S. Hemelryk, G. Falony, and L. De Vuyst, "Volatile analysis of spoiled, artisan-type, modifiedatmosphere-packaged cooked ham stored under different temperatures," Food Microbiology, pp. 94-102, 2009.

[25] A. Loutfi, S. Coradeschi, G. Mani, P. Shankar, B. Rayappan, and J. Bosco, "Electronic noses for food quality: a review," Journal of Food Engineering, vol. 144, pp. 103-111, 2015.

[26] J. Liu, X. Huang, G. Ye, L. Wei, Z. Jiao, W. Chao et al., "H2S detection sensing characteristic of $\mathrm{CuO} / \mathrm{SnO}_{2}$ sensor," Sensors, vol. 2, pp. 110-118, 2003. 

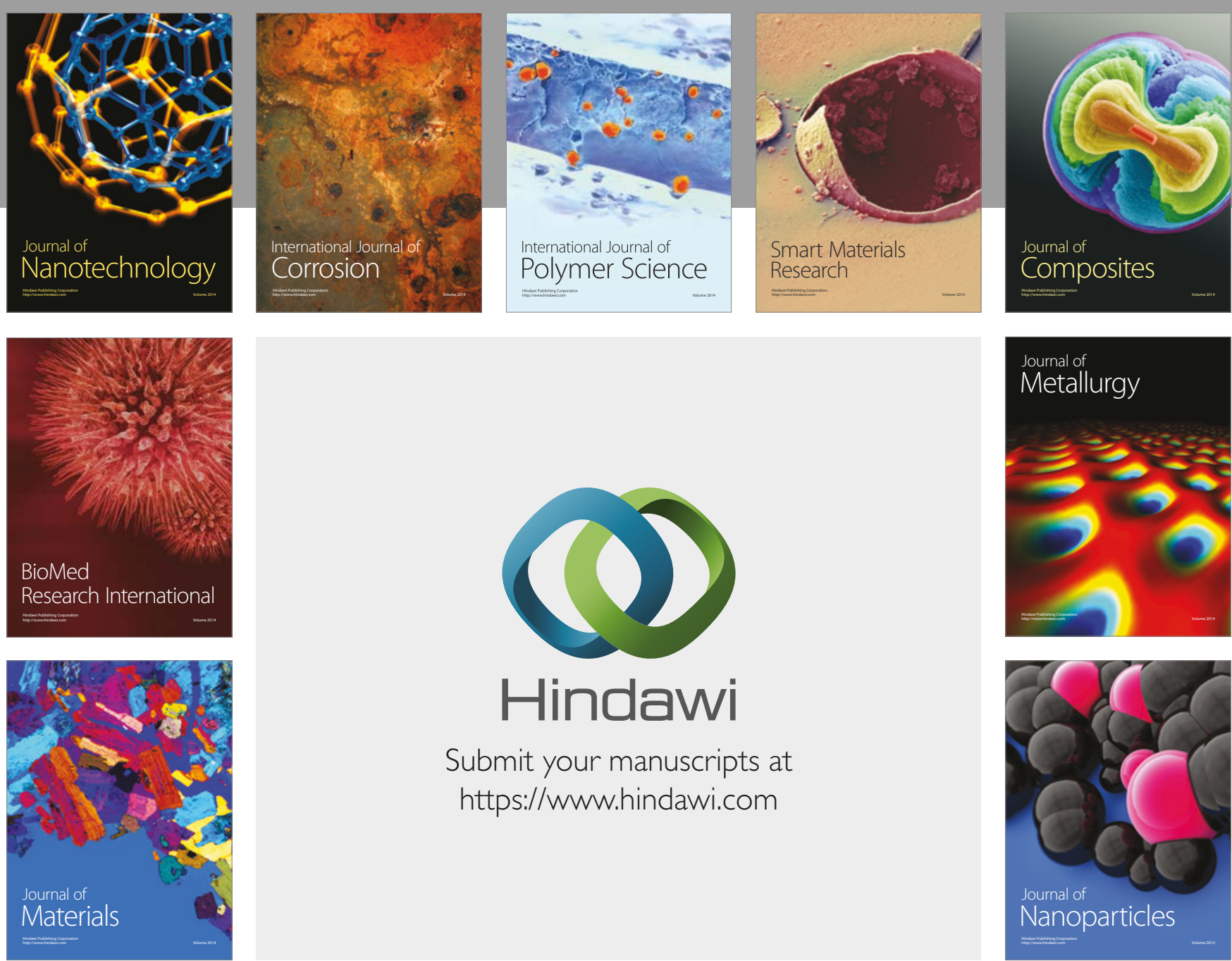

\section{Hindawi}

Submit your manuscripts at

https://www.hindawi.com
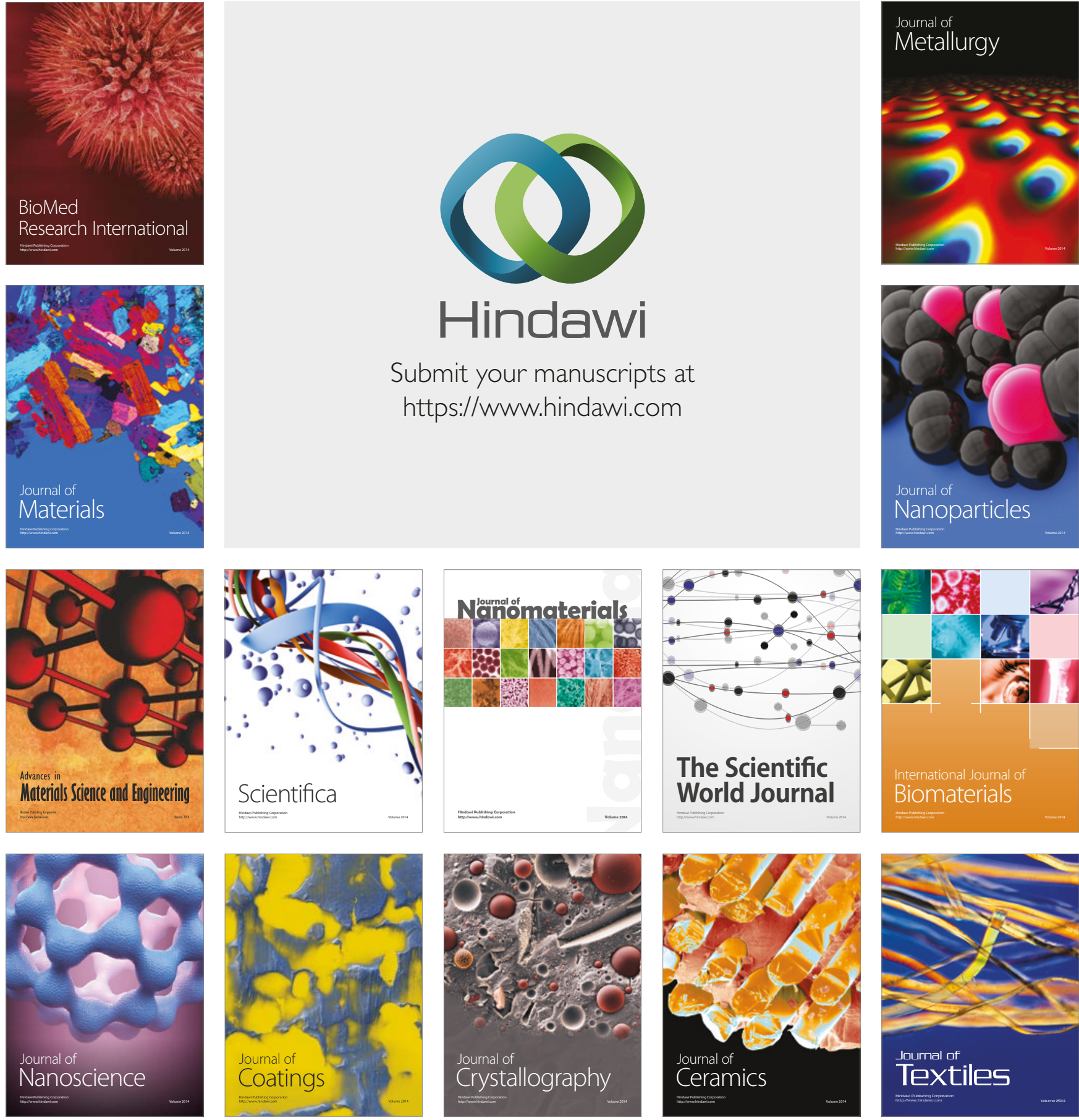

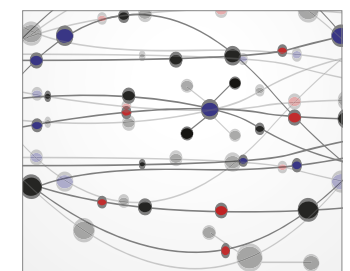

The Scientific World Journal
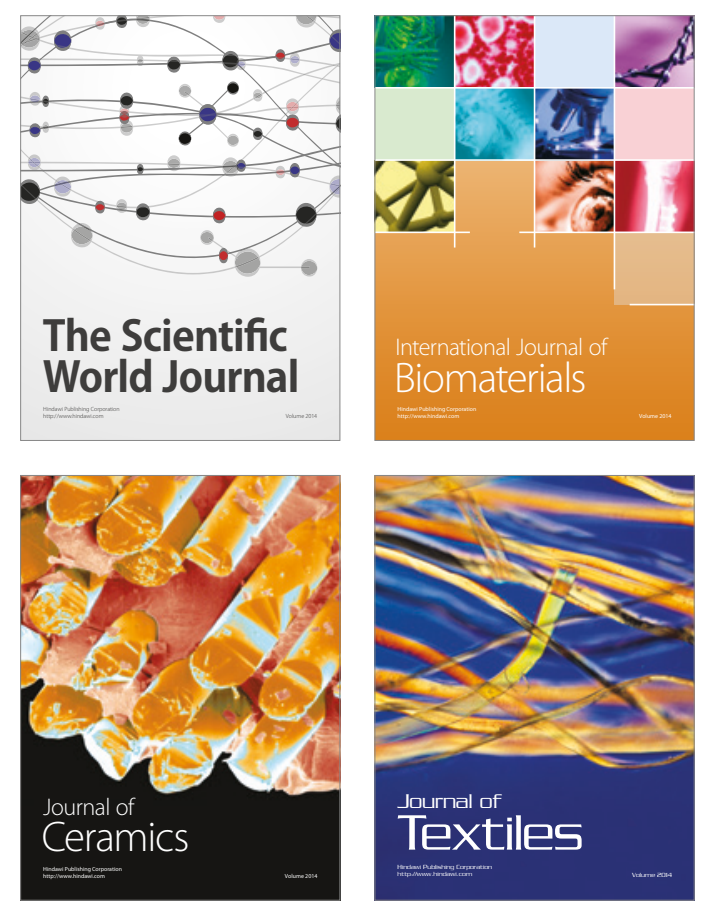